\title{
Strategies to enhance paracrine potency of transplanted mesenchymal stem cells in intractable neonatal disorders
}

\author{
Won Soon Park ${ }^{1,3}$, So Yoon Ahn ${ }^{1,3}$, Se In Sung ${ }^{1}$, Jee-Yin $\mathrm{Ahn}^{2}$ and Yun Sil Chang ${ }^{1}$
}

Mesenchymal stem cell (MSC) transplantation represents the next breakthrough in the treatment of currently intractable and devastating neonatal disorders with complex multifactorial etiologies, including bronchopulmonary dysplasia, hypoxic ischemic encephalopathy, and intraventricular hemorrhage. Absent engraftment and direct differentiation of transplanted MSCs, and the "hit-and-run" therapeutic effects of these MSCs suggest that their pleiotropic protection might be attributable to paracrine activity via the secretion of various biologic factors rather than to regenerative activity. The transplanted MSCs, therefore, exert their therapeutic effects not by acting as "stem cells," but rather by acting as "paracrine factors factory." The MSCs sense the microenvironment of the injury site and secrete various paracrine factors that serve several reparative functions, including antiapoptotic, anti-inflammatory, antioxidative, antifibrotic, and/or antibacterial effects in response to environmental cues to enhance regeneration of the damaged tissue. Therefore, the therapeutic efficacy of MSCs might be dependent on their paracrine potency. In this review, we focus on recent investigations that elucidate the specifically regulated paracrine mechanisms of MSCs by injury type and discuss potential strategies to enhance paracrine potency, and thus therapeutic efficacy, of transplanted MSCs, including determining the appropriate source and preconditioning strategy for MSCs and the route and timing of their administration.

D espite recent advances in neonatal intensive care medicine, intractable neonatal disorders, including bronchopulmonary dysplasia (BPD) $(1,2)$, severe intraventricular hemorrhage (IVH) (3), and hypoxic ischemic encephalopathy (HIE) (4), remain major causes of mortality and serious morbidities in survivors. Currently, few effective therapies are available to ameliorate injuries resulting from these disorders. Therefore, the development of new, safe, and effective therapies to improve the outcomes of these devastating neonatal disorders is an urgent issue.
Recently, several preclinical studies have demonstrated the promise of stem cell therapies in attenuating tissue injuries in newborn animal models of BPD (5-10), HIE (11), and IVH (12-15). Furthermore, phase I clinical studies in newborn infants with BPD (16), HIE (17), or severe IVH have shown that stem cell treatments for newborn infants might be safe, feasible, and potentially efficacious. Taken together, these findings suggest that stem cells might represent a paradigm shift in the treatment of currently intractable and devastating neonatal disorders. However, stem cell therapies are still experimental, and the precise mechanisms of action underlying them remain to be elucidated. This review summarizes the therapeutic potential of stem cells for these neonatal disorders. We focus on the paracrine protective mechanism underlying the beneficial effects of stem cell therapies and potential strategies to enhance the paracrine potency, and thus therapeutic efficacy of MSC transplantation to facilitate bench-to-bedside translation of stem cell therapies for these disorders.

\section{PROTECTIVE MECHANISMS UNDERLYING STEM CELL THERAPIES \\ Pleiotropic Protective Effects of Stem Cell Therapies}

Because the pathophysiological mechanisms of tissue injuries after BPD (18-21), severe IVH $(22,23)$, or HIE $(24)$ are complex and multifactorial, modulating only one factor might not be sufficient to ameliorate the disease. Therefore, a multifaceted therapeutic agent might be necessary to improve outcomes of patients with these intractable neonatal disorders. The pleiotropic beneficial effects of stem cell therapy, such as antiapoptotic, anti-inflammatory, antifibrotic, and antioxidative effects, have been observed in various animal models of BPD (5-8,10), severe IVH $(13-15)$, or HIE $(11,25,26)$. Furthermore, in addition to their beneficial antiinflammatory effects, antibacterial activity of transplanted stem cells were observed in an animal model of Escherichia coli pneumonia (27). Considering their manifold therapeutic effects, stem cells, rather than other single therapeutic agents, might be the most promising candidates for therapies aimed at improving the prognosis of certain neonatal disorders.

\footnotetext{
${ }^{1}$ Samsung Medical Center, Sungkyunkwan University School of Medicine, Seoul, South Korea; ${ }^{2}$ Department of Molecular Cell Biology, Sungkyunkwan University School of Medicine, Suwon, South Korea. Correspondence: Yun Sil Chang or Jee-Yin Ahn (cys.chang@samsung.com or jeeahn@skku.edu)

${ }^{3}$ These authors contributed equally to this work as co-first authors.

Received 25 May 2017; accepted 21 September 2017; advance online publication 1 November 2017. doi:10.1038/pr.2017.249
} 


\section{Engraftment and Regeneration}

Recent insights into the biology of stem cells have ignited the hope of regenerating damaged organs by stem cell transplantation (28-34). Among various stem cell therapies $(35,36)$, mesenchymal stem cells (MSCs) have emerged as the most promising therapeutic candidates in regenerative medicine, as they are more ethically and socially acceptable and show less tumorigenicity than embryonic stem cells. Because of their multilineage differentiation potential, the protective effects of MSC transplantation were initially ascribed to the engraftment of these cells in injured tissues and their subsequent transdifferentiation to repair and replace damaged cells $(37,38)$. However, the very low rate of in vivo engraftment and differentiation of transplanted MSCs $(8,15,39)$ suggests that long-term survival of MSCs might not be essential for their beneficial effects $(28,40)$. Therefore, the therapeutic effects of MSC transplantation might not be associated with their differentiation and direct replenishment of damaged tissue parenchymal cells.

\section{Paracrine Protection}

Not only MSCs but also their conditioned media were able to ameliorate hyperoxia-induced $(41,42)$ or lipopolysaccharideinduced (43) acute lung injuries. Moreover, therapeutic efficacy of other cell types, including endothelial progenitor and amniotic epithelial cells, in BPD has also been reported $(10,44,45)$. These findings suggest that the protective mechanisms of MSC transplantation might mainly be related to their ability to stimulate the survival and recovery of damaged tissue by paracrine manners (Figure 1).

In various tissue injury models, transplanted MSCs exert anti-inflammatory, antifibrotic, antioxidative, antiapoptotic, antimicrobial, and permeability-decreasing paracrine effects via secretion of soluble factors. These soluble factors include various cytokines such as transforming growth factor- $\beta$ (46) and interleukin-10 (ref. 47), growth factors such as vascular endothelial growth factor (48), hepatocyte growth factor (49), keratinocyte growth factor (50) brain-derived neutrophic factor $(13,51)$, nerve growth factors (51), and neurotrophin-3 (ref. 51) insulin growth factor-1 (ref. 52), proteins such as angiopietin-1 (ref. 53), tumor necrosis factor-stimulated gene 6 (ref. 54), interleukin-1 receptor antagonist (55), lipocalin-2 (ref. 56) LL-37 (ref. 57), defensin-2 (ref. 58) and others. Recent reports also demonstrated that MSCs ameliorated and resolved inflammation by producing proresolving lipid mediators such as lipoxin A4 in acute lung injury (59) and resolvins in animal models of sepsis (60).

Recently, in addition to the cytokines and other secreted molecules mentioned above, MSC derived-extracellular vesicles (EVs) or exosomes $(28,40,61-63)$ were shown to be key mediators of MSC therapeutic action (62-64). EVs derived from MSCs show therapeutic effects on various tissue injury in preclinical animal models by modulating immune response (65), ameliorating oxidative stress (66), and decreasing apoptosis (67), which is similar to what is achieved using the originating MSCs themselves. In recent studies using newborn animals, MSC-derived EVs protected neonatal lungs after hyperoxic injury (68), fetal brains after hypoxiaischemia (64), and the intestine from experimental necrotizing enterocolitis (69).

The therapeutic mechanism of MSC-derived EVs or exosomes has been known to be related to the transfer of their vesicular cargo molecules, which mediate cell-to-cell communication. These vesicular molecules are biologically active and include proteins, RNAs such as messenger RNA, microRNA, and transfer RNA, as well as bioactive lipids $(70,71)$. Detailed information regarding MSC-derived EVs or exosomes can be found in other focused reviews $(72,73)$.

However, as a paracrine mechanism of action of MSCs, the importance of the presence of MSCs themselves rather than the MSC secretome for early recovery from ventilator-induced lung injury has been highlighted (74). In addition, attachment of live MSCs to the alveolar epithelium in acute lung injury was shown to be critical for mitochondrial transfer of MSCs (75).

Recently, transfer of mitochondria from MSCs has been demonstrated to be pivotal for the beneficial effects of MSCs (76-78). Recent reports showed that mitochondrial transfer occurred from MSCs to macrophages (78), partly through tunneling nanotubes (77), and the transfer of functional mitochondria in EVs is responsible for the anti-inflammatory effects of MSCs on macrophages in the inflammatory milieu $(72,79)$.

Taken together, these findings suggest that the pleiotropic protective effects of transplanted stem cells might be mediated predominantly by paracrine action via the secretion of various biologic factors-a "hit-and-run" mechanism $(80,81)$-rather than by direct regenerative action (82-85). The use of a cellfree preparation comprising MSC-derived EVs or exosomes in place of stem cells shows excellent promise as a new therapeutic approach for neonatal disorders, as it circumvents side effects such as tumor formation that are associated with treatments with live stem cells.

Environmental Cues Trigger the Secretion of Paracrine Factors Accumulating evidence indicates that MSCs release cytoprotective paracrine factors strictly in response to environmental cues $(86,87)$. We observed that despite the use of the same human umbilical cord blood (UCB)-derived MSCs, pivotal cytoprotective paracrine factors varied by disease animal model. The protective effects of human UCB-derived MSC transplantation on hyperoxic neonatal lung injuries were associated with significant upregulation of hyperoxia-induced vascular endothelial growth factor (VEGF) and hepatocyte growth factor (11). Furthermore, knockdown of VEGF secreted by MSCs (7) transfected with small interfering RNAs specific for human VEGF abolished the protective effects of MSCs, such as attenuation of impaired alveolarization and angiogenesis, reduction of increased terminal deoxynucleotidyl transferase nick-end labeling - and ED-1-positive cells, and downregulation of proinflammatory cytokine expression, indicating that VEGF secreted by transplanted MSCs (7) and 


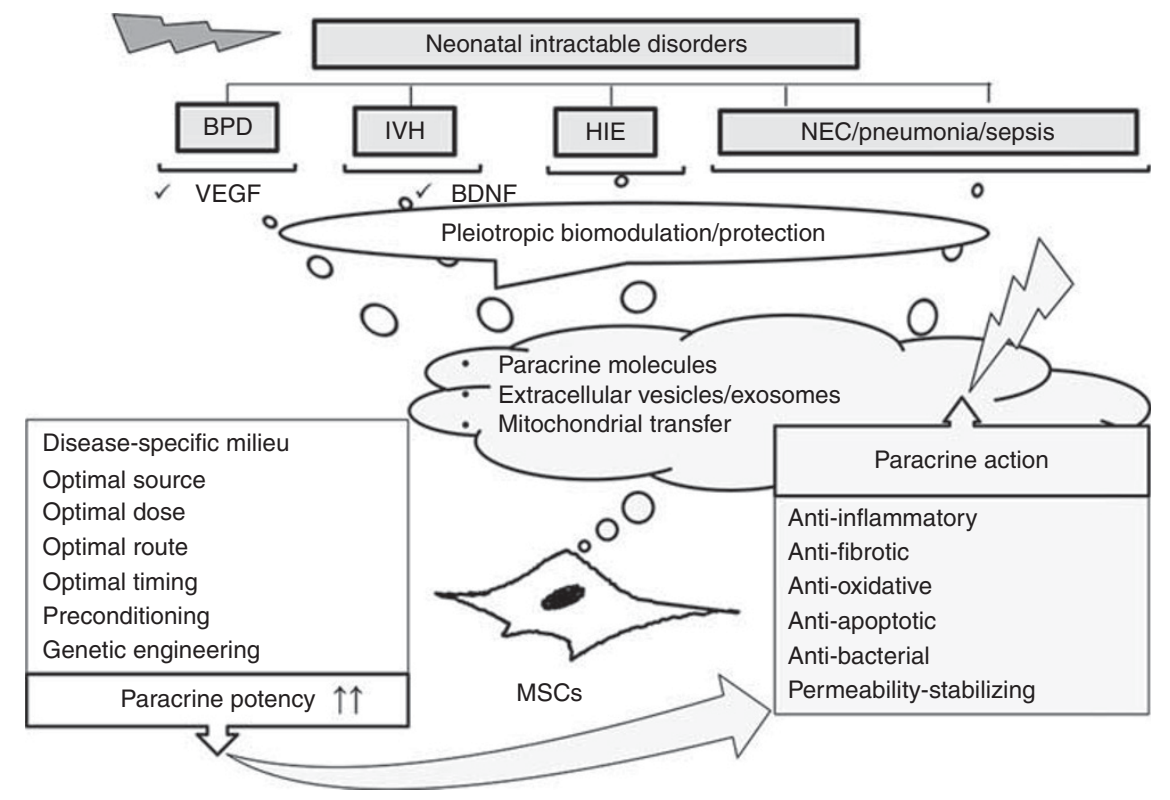

Figure 1. Schematic showing paracrine mechanism of pleiotropic biomodulation and protection provided by mesenchymal stem cells (MSCs) against neonatal intractable disorders such as bronchopulmonary dysplasia (BPD), intraventricular hemorrhage (IVH), hypoxic-ischemic encephalopathy (HIE), and others. Phase I clinical trials in newborn infants have shown that stem cell transplantation is safe and feasible in BPD, IVH, and HIE. Several preclinical evidences of protection against other diseases such as necrotizing enterocolitis (NEC), pneumonia, and sepsis post-MSC transplantation or application of MSC-secreted extracellular vesicles or exosomes in animal models are available. MSCs locally or systemically transplanted into various tissue injuries exert anti-inflammatory, antifibrotic, antioxidative, antiapoptotic, antibacterial, and permeability-stabilizing paracrine actions through the secretion of soluble paracrine molecules. Among them, MSC-derived extracellular vesicles or exosomes are known as the key mediators of MSC therapeutic effects. Moreover, transfer of mitochondria from MSCs to host cells is one of the important paracrine mechanisms underlying the beneficial effects of MSCs. The paracrine potency of MSCs are associated with disease-specific environmental milieu, and potential strategies for enhancing the paracrine potency of MSCs include optimal determination of cell source, dose, route, and timing for MSC transplantation. Furthermore, various preconditioning and genetic engineering of MSCs can potentiate the paracrine potency of MSCs. MSC-secreted vascular endothelial growth factor (VEGF) and brain-derived neurotrophic factor (BDNF) are critical paracrine factors mediating protection obtained after MSC transplantation against BPD and severe IVH, respectively.

contained within MSC-derived exosomes (not yet published) is a critical paracrine factor that plays seminal roles in attenuating hyperoxic neonatal lung injuries. Although the same human UCB-derived MSCs as those used to treat neonatal hyperoxic lung injuries were transplanted into a newborn animal model of severe IVH, we observed significant upregulation of brain-derived neurotrophic factor (BDNF) both in DNA and antibody microarray analyses (13). Furthermore, in newborn rats with severe IVH, knockdown of BDNF secreted by MSCs abolished the neuroprotective effects of MSCs, such as significantly reduced posthemorrhagic hydrocephalus and impaired behavior, increased apoptosis, inflammation, and astrogliosis, and reduced myelination, indicating that BDNF secreted by transplanted MSCs is a critical paracrine factor that plays critical roles in attenuating severe IVH-induced brain injuries in neonatal rats. Additionally, Toll-like receptor-4 (TLR-4) signaling in transplanted MSCs and the subsequent secretion of $\beta$ defensin 2 was essential in mediating the antibacterial and anti-inflammatory protective effects of MSCs in acute lung injuries following E. coli-induced pneumonia (27). Our conflicting data on the critical role of the proinflammatory phenotype of TLR-4-primed MSCs (88), which exerted antiinflammatory properties in E. coli-induced acute lung injuries, suggest that MSCs sense and control host inflammation by switching between their roles as proinflammatory or antiinflammatory mediators (89). In addition, TLR-4 in MSCs plays pivotal roles in eliminating pathogens by augmenting antibacterial effects and reducing host tissue injuries by attenuating the inflammatory response $(90,91)$. Collectively, these studies suggest that key paracrine factors secreted by MSCs from the same source play important roles in mediating the therapeutic effects of MSCs in different preclinical disease models $(11,13,48)$, suggesting that there is a crosstalk and interplay between the host tissue and transplanted MSCs $(61,92)$. Therefore, unlike drug treatments that deliver a single agent at a specific dose, transplanted MSCs act as a "paracrine factors factory" that sense the microenvironment of the injury site and secrete various paracrine factors that exert several reparative functions, including antiapoptotic, anti-inflammatory, antioxidative, antifibrotic, and/or antibacterial effects in response to local microenvironmental cues to enhance the regeneration of damaged tissue (86).

Moreover, recently, the change of the name of MSCs into "medicinal signaling cells" were proposed instead of calling as "stem cells" because transplanted MSCs to treat the diseases act their primary beneficial and medicinal function at the injury sites of the body through their secretory action (93). 
POTENTIAL STRATEGIES TO ENHANCE PARACRINE POTENCY OF STEM CELL THERAPIES

As the therapeutic efficacy of MSCs seems to be dependent on the paracrine potency of MSCs, the following potential strategies to enhance the paracrine potency of MSCs, including determining the best source, route, timing, and preconditioning approach for MSCs, might improve the therapeutic efficacy of transplanted MSCs $(9,35,82,84,94,95)$.

\section{Paracrine Potency Assay}

As implied by the relatively loose minimal criteria for defining MSCs, including their fibroblast-like morphology, plastic adherence in culture, defined cell surface marker expression profiles (CD 73-, 90-, and 105-positive and CD 45-, 34-, 14-, and 11b-negative), and capacity for differentiation into cell types such as adipocytes, chondrocytes, and osteoblasts (96), MSCs might represent a heterogeneous cell population $(83,96)$. Therefore, their paracrine potency and therapeutic efficacy might vary with source (97-99) and batch (100) of MSCs. Therefore, identifying a specific marker or feature to predict the in vivo therapeutic potential of transplanted MSCs is the Holy Grail in clinical translation of MSCs transplantation for use in neonatal disorders $(101,102)$.

The close association of the therapeutic efficacy of MSCs with their paracrine potency suggests that measuring paracrine potency of MSCs might be a surrogate measure of their in vivo therapeutic efficacy. The expression of soluble tumor necrosis factor receptor-1 in MSCs was quantitatively assayed as a surrogate measure of potency for the treatment of steroid-resistant acute graft-vs-host disease (103). However, as the clinical trial failed to meet the primary criterion of therapeutic efficacy, it remains unknown whether soluble tumor necrosis factor receptor-1 by MSC expression levels are predictive of the in vivo therapeutic efficacy of MSCs. An in vitro assay measuring IL-10 released from blood cells might be useful in analyzing the potency of MSC-conditioned media and MSC lysates (104). Our data indicating a critical role for VEGF secreted by MSCs in BPD (7) and in BDNF and severe IVH (13) suggests that quantification of these factors might be used as a potency biomarker assay to select MSCs with the best predicted in vivo therapeutic efficacy for application in neonatal disorders. Further studies will be necessary to identify robust and predictive markers of therapeutic efficacy and develop quantitative assays that measure the paracrine potency of transplanted MSCs and predict their in vivo efficacy.

\section{Optimal Cell Source}

MSCs obtained from gestational tissues such as UCB (105), Wharton's jelly, or umbilical cords (106) showed increased secretion of chemokines, proinflammatory proteins, and growth factors, as well as higher rate of cell proliferation, than MSCs obtained from adult adipose tissue or bone marrow. Furthermore, in our recent study comparing the in vivo therapeutic efficacy of adipose tissue- and UCBderived MSCs and UCB-derived mononuclear cells in protecting against hyperoxic lung injuries in newborn rats, UCB-derived MSCs exhibited better therapeutic efficacy in attenuating hyperoxic lung injuries, with effects such as impaired alveolarization and angiogenesis, increased cell death, alveolar macrophages, and proinflammatory cytokines, and increased secretion of VEGF and hepatocyte growth factor, compared with that of adipose tissue MSCs or UCBderived mononuclear cells (10). Collectively, as donor age negatively impacts the paracrine potency of stem cells and thus the therapeutic efficacy of stem cell therapies, birthassociated tissues such as UCB or Wharton's jelly might be the optimal source for MSCs in future clinical applications to protect against intractable neonatal disorders (107).

\section{Preconditioning of Stem Cells}

There is growing evidence that in vitro preconditioning of MSCs can optimize their paracrine potency and thus their therapeutic potential $(82,108-110)$. Preconditioning of MSCs includes exposure of in vitro MSCs to hypoxic or anoxic conditions (111-114); e.g., the addition of growth factors such as epidermal growth factor (115), glial cell-derived neurotrophic factor (116), insulin-like growth factor-1 (ref. 117); cytokines such as tumor necrosis factor- $\alpha$ (118), or stromal cell-derived factor-1 (ref. 119); hormones such as angiotensinII (ref. 116), melatonin (120), or lipopolysaccharides (121); and pharmacologic or chemical agents such as hydrogen peroxide (122), deferoxamine (123), or diazoxide (124). Although manifold mechanisms, including improved in vivo survival and engraftment after transplantation (125), might be involved, enhancement of the therapeutic potential of MSCs by preconditioning seems to be mediated primarily by stimulating the secretion of growth factors, cytokines, and other proteins, as well as the release of exosomes and EVs from MSCs (114). Enhanced secretion of paracrine factors by preconditioned MSCs has various trophic, immunomodulatory, antiapoptotic, and proangiogenic effects (82). However, the paracrine profiles of the secretomes obtained from preconditioned MSCs are known to vary according to the preconditioning regimen used. Therefore, although encouraging preclinical data increases the hope that preconditioning can enhance the reparative and regenerative capacities of MSCs, additional comprehensive studies will be necessary to decipher the whole secretomes of MSCs, including exosomes secreted after different preconditioning regimens (125), and, based on these data, to establish the optimal preconditioning regimen and schedule to ensure maximal paracrine potency and therapeutic efficacy of transplanted MSCs.

\section{Genetic Engineering of Stem Cells}

One promising therapeutic strategy is enhancing the release of a specific paracrine regenerative factor from stem cells by overexpressing it through genetic engineering (108). In agreement with this hypothesis, overexpression of VEGF in MSCs enhanced stem cell-mediated therapeutic efficacy in neural and cardiac repair (126), and intracerebral transplantation of BDNF gene-modified MSCs 1 day after middle 


\section{Review | Park et al.}

cerebral artery occlusion promoted functional recovery and reduced infarct sizes in rats (127). In contrast, intranasal transplantation of BDNF-overexpressing MSCs 3 days after neonatal stroke failed to promote recovery from middle cerebral artery occlusion-induced brain injuries (128). Overall, these findings suggest that several details, including route, timing, and dose, might be confounding variables that determine the success or failure of transplanting genetically engineered MSCs. Further studies will be necessary to confirm this. Apart from therapeutic efficacy, safety issues are major limitations to future clinical therapeutic applications of genetically engineered MSCs, as viral integration in the host genome increases tumorigenicity (129). Although the risk of tumorigenicity can be reduced by using an adenovirus vector that does not integrate into the host genome instead of lentior retroviruses, further studies that closely monitor the fate of transplanted gene-modified MSCs will be necessary to address these safety concerns.

\section{Optimal Route of Administration}

Determining the optimal route for stem cell transplantation might be a critical issue for clinical translation of this therapy. Systemically or intravenously administered MSCs are known to migrate and localize chemotactically to injury sites in host tissue $(34,130)$. Furthermore, systemically injected MSCs are usually retained mainly in the lungs and other organs such as liver, spleen, and kidneys $(42,131)$. In addition, systemic intravenous or intraperitoneal routes of MSC transplantation are less invasive and might be more suitable for use in unstable newborn infants than the more invasive local route of administration such as intratracheal or intracerebroventricular injection. However, systemically transplanted MSCs have the disadvantage of nonspecific targeting because of their broad dissemination and ability to cross an intact blood-brain barrier. In our previous studies, although four- to fivefold higher doses of MSCs were administered intravenously or intraperitoneally (systemically) than administered intratracheally or intraventricularly (locally), significantly higher numbers of donor MSCs were correctly localized in the lungs or brains of a newborn rat model of hyperoxia (5) or severe IVH (14), respectively. Moreover, local, rather than systemic, transplantation of MSCs was associated with greater paracrine potency in the production of trophic factors such as VEGF and hepatocyte growth factor (11), and thus better therapeutic efficacy against newborn BPD (5) and severe IVH (14) in animal models. Paracrine signals are transmitted only over short distances via factors that exert local effects (82). The cross-talk between the microenvironment of injured host tissues and MSCs activates MSCs to produce cytoprotective paracrine factors. Therefore, proximity of the donor cells to the injury site is essential for their paracrine-protective effects. Collectively, these findings support the assumption that local, rather than systemic, administration of MSCs might be the optimal route for MSC transplantation to enhance tissue repair.

\section{Determining the Optimal Timing}

Determining the optimal timing for MSC transplantation is another important factor in clinical translation of this therapy. Although the therapeutic windows for MSC transplantation to address neonatal disorders vary widely according to the animal model used and the severity of tissues injury, early rather than late transplantation of MSCs better attenuated hyperoxic lung injuries (6), severe IVH (132), neonatal stroke (133), and hypoxic-ischemic encephalopathy (11). Moreover, our data on upregulation of growth factors such as VEGF and hepatocyte growth factor with early but not with late transplantation of MSCs, despite higher donor cell localization in neonatal hyperoxic lung injuries (6), suggest that the protective effects of MSCs, including proangiogenic, antioxidative, anti-inflammatory, antifibrotic, and antiapoptotic effects, might be associated with or mediated by enhanced secretion of these paracrine growth factors (134). In contrast, prolonged survival and engraftment of donor cells might not be essential for their paracrine potency $(28,40)$. Moreover, as inflammation might affect the secretion of these growth factors by MSCs (135), MSC transplantation soon after injury might be essential for their paracrine potency and the resultant therapeutic efficacy. Overall, the therapeutic time window for stem cell therapy might be narrow, and MSC transplantation as close as possible to the time of brain insult might be optimal for increased paracrine potency and therapeutic outcomes. Further studies will be necessary to confirm this.

\section{Determining the Optimal Dose}

Considering various preclinical data showing wide variations in the paracrine potency and therapeutic efficacy of transplanted MSCs according to the timing and route of stem cell administration in different animal models of BPD $(6,11)$, HIE (136), and IVH (14), optimal doses need to be determined at the specific injury site and with the specific timing and route of MSC transplantation.

\section{CONCLUSION}

Recent preclinical and clinical studies suggest that MSC transplantation could be a game changer for treating currently intractable neonatal disorders with complex multifactorial etiologies, including BPD, HIE, and IVH. MSCs act as "paracrine factors factory" by secreting various paracrine factors are responsible for their pleiotropic effects in response to microenvironmental cues in the injured host tissues. Therefore, MSC transplantation might open a new chapter in tailor-made neonatal medicine. However, further meticulous studies to delineate the paracrine-protective mechanisms of MSCs with specific injuries and to determine strategies, including the best source, preconditioning regimen, route, timing, and dose of transplanted MSCs, to enhance the paracrine potency and, thus, the therapeutic efficacy of MSCs will be necessary for successful clinical translation of these therapies. 


\section{Enhancing paracrine potency of MSCs $\mid$ Review}

\section{ACKNOWLEDGMENTS}

This work was supported by grant Samsung Biomedical Research Institute grant (SMX1170321) and by grant from the Korea Health Technology R\&D Project through the Korea Health Industry Development Institute (KHIDI), funded by the Ministry of Health and Welfare, Republic of Korea (HI14C3484).

Disclosure: Won Soon Park and Yun Sil Chang declare potential conflicts of interest arising from a filed or issued patent titled "Composition for treating intraventricular hemorrhage in preterm infants comprising mesenchymal stem cells" and "Method of treating lung diseases using cells separated or proliferated from umbilical cord blood" as coinventors, not as patentees.

\section{REFERENCES}

1. Bland RD. Neonatal chronic lung disease in the post-surfactant era. Biol Neonate 2005;88:181-91.

2. Bhandari A, Panitch HB. Pulmonary outcomes in bronchopulmonary dysplasia. Semin Perinatol 2006;30:219-6.

3. Vohr BR, Wright LL, Dusick AM, et al. Neurodevelopmental and functional outcomes of extremely low birth weight infants in the National Institute of Child Health and Human Development Neonatal Research Network, 1993-1994. Pediatrics 2000;105:1216-26.

4. Johnston MV. Hypoxic and ischemic disorders of infants and children. Lecture for 38th meeting of Japanese Society of Child Neurology, Tokyo, Japan, July 1996. Brain Dev 1997;19:235-9.

5. Chang YS, Oh W, Choi SJ, et al. Human umbilical cord blood-derived mesenchymal stem cells attenuate hyperoxia-induced lung injury in neonatal rats. Cell Transplant 2009;18:869-86.

6. Chang YS, Choi SJ, Ahn SY, et al. Timing of umbilical cord blood derived mesenchymal stem cells transplantation determines therapeutic efficacy in the neonatal hyperoxic lung injury. PLoS ONE 2013;8:e52419.

7. Chang YS, Ahn SY, Jeon HB, et al. Critical role of vascular endothelial growth factor secreted by mesenchymal stem cells in hyperoxic lung injury. Am J Respir Cell Mol Biol 2014;51:391-9.

8. Ahn SY, Chang YS, Kim SY, et al. Long-term (postnatal day 70) outcome and safety of intratracheal transplantation of human umbilical cord blood-derived mesenchymal stem cells in neonatal hyperoxic lung injury. Yonsei Med J 2013;54:416-24.

9. Ahn SY, Chang YS, Park WS. Stem cell therapy for bronchopulmonary dysplasia: bench to bedside translation. J Korean Med Sci 2015;30: 509-13.

10. Ahn SY, Chang YS, Sung DK, et al. Cell type-dependent variation in paracrine potency determines therapeutic efficacy against neonatal hyperoxic lung injury. Cytotherapy 2015;17:1025-35.

11. Sung DK, Chang YS, Ahn SY, et al. Optimal route for human umbilical cord blood-derived mesenchymal stem cell transplantation to protect against neonatal hyperoxic lung injury: gene expression profiles and histopathology. PLoS ONE 2015;10:e135574.

12. Ahn SY, Chang YS, Park WS. Mesenchymal stem cells transplantation for neuroprotection in preterm infants with severe intraventricular hemorrhage. Korean J Pediatr 2014;57:251-6.

13. Ahn SY, Chang YS, Sung DK, Sung SI, Ahn JY, Park WS. Pivotal role of brain derived neurotrophic factor secreted by mesenchymal stem cells in severe intraventricular hemorrhage in the newborn rats. Cell Transplant 2016;26:145-56.

14. Ahn SY, Chang YS, Sung DK, et al. Optimal route for mesenchymal stem cells transplantation after severe intraventricular hemorrhage in newborn rats. PLoS ONE 2015;10:e0132919.

15. Ahn SY, Chang YS, Sung DK, et al. Mesenchymal stem cells prevent hydrocephalus after severe intraventricular hemorrhage. Stroke 2013;44: 497-504.

16. Chang YS, Ahn SY, Yoo HS, et al. Mesenchymal stem cells for bronchopulmonary dysplasia: phase 1 dose-escalation clinical trial. J Pediatr 2014;164:966-72 e6.
17. Cotten CM, Murtha AP, Goldberg RN, et al. Feasibility of autologous cord blood cells for infants with hypoxic-ischemic encephalopathy. J Pediatr 2014;164:973-9 e1.

18. Popova AP. Mechanisms of bronchopulmonary dysplasia. J Cell Commun Signal 2013;7:119-27.

19. Jobe AH, Bancalari E. Bronchopulmonary dysplasia. Am J Respir Crit Care Med 2001;163:1723-9.

20. Stenmark KR, Abman SH. Lung vascular development: implications for the pathogenesis of bronchopulmonary dysplasia. Annu Rev Physiol 2005;67:623-1.

21. Baraldi E, Filippone M. Chronic lung disease after premature birth. N Engl J Med 2007;357:1946-55.

22. Ballabh P. Intraventricular hemorrhage in premature infants: mechanism of disease. Pediatr Res 2010;67:1-8.

23. Ballabh P. Pathogenesis and prevention of intraventricular hemorrhage. Clin Perinatol 2014;41:47-67.

24. Calvert JW, Zhang JH. Pathophysiology of an hypoxic-ischemic insult during the perinatal period. Neurol Res 2005;27:246-60.

25. Gonzales-Portillo GS, Reyes S, Aguirre D, Pabon MM, Borlongan CV. Stem cell therapy for neonatal hypoxic-ischemic encephalopathy. Front Neurol 2014;5:147.

26. Pimentel-Coelho PM, Mendez-Otero R. Cell therapy for neonatal hypoxic-ischemic encephalopathy. Stem Cells Dev 2010;19:299-310.

27. Kim ES, Chang YS, Choi SJ, et al. Intratracheal transplantation of human umbilical cord blood-derived mesenchymal stem cells attenuates Escherichia coli-induced acute lung injury in mice. Respir Res 2011;12: 108.

28. van Poll D, Parekkadan B, Cho $\mathrm{CH}$, et al. Mesenchymal stem cellderived molecules directly modulate hepatocellular death and regeneration in vitro and in vivo. Hepatology 2008;47:1634-43.

29. van Velthoven CT, Kavelaars A, van Bel F, Heijnen CJ. Mesenchymal stem cell transplantation changes the gene expression profile of the neonatal ischemic brain. Brain Behav Immun 2011;25:1342-8.

30. Zisa D, Shabbir A, Suzuki G, Lee T. Vascular endothelial growth factor (VEGF) as a key therapeutic trophic factor in bone marrow mesenchymal stem cell-mediated cardiac repair. Biochem Biophys Res Commun 2009;390:834-.

31. Abman SH, Matthay MA. Mesenchymal stem cells for the prevention of bronchopulmonary dysplasia: delivering the secretome. Am J Respir Crit Care Med 2009;180:1039-41.

32. Kunter U, Rong S, Djuric Z, et al. Transplanted mesenchymal stem cells accelerate glomerular healing in experimental glomerulonephritis. J Am Soc Nephrol 2006;17:2202-12.

33. Zappia E, Casazza S, Pedemonte E, et al. Mesenchymal stem cells ameliorate experimental autoimmune encephalomyelitis inducing T-cell anergy. Blood 2005;106:1755-61.

34. Ortiz LA, Gambelli F, McBride C, et al. Mesenchymal stem cell engraftment in lung is enhanced in response to bleomycin exposure and ameliorates its fibrotic effects. Proc Natl Acad Sci USA 2003;100: 8407-11.

35. Park WS, Ahn SY, Sung SI, Ahn JY, Chang YS, , . Mesenchymal stem cells: the magic cure for intraventricular hemorrhage? Cell Transplant 2017;26:439-8.

36. Lekic T, Manaenko A, Rolland W, Tang J, Zhang JH. A novel preclinical model of germinal matrix hemorrhage using neonatal rats. Acta Neurochir Suppl 2011;111:55-60.

37. Bussolati B. Stem cells for organ repair: support or replace? Organogenesis 2011;7:95.

38. Togel F, Westenfelder C. Adult bone marrow-derived stem cells for organ regeneration and repair. Dev Dyn 2007;236:3321-1.

39. Godfrey KM, James MP. Treatment of severe acne with isotretinoin in patients with inflammatory bowel disease. Br J Dermatol 1990;123: 653-5.

40. Parekkadan B, van Poll D, Suganuma K, et al. Mesenchymal stem cellderived molecules reverse fulminant hepatic failure. PLoS One 2007;2: e941. 
41. Pierro M, Ionescu L, Montemurro T, et al. Short-term, long-term and paracrine effect of human umbilical cord-derived stem cells in lung injury prevention and repair in experimental bronchopulmonary dysplasia. Thorax 2012;68:475-84.

42. Aslam M, Baveja R, Liang OD, et al. Bone marrow stromal cells attenuate lung injury in a murine model of neonatal chronic lung disease. Am J Respir Crit Care Med 2009;180:1122-30.

43. Ionescu L, Byrne RN, van Haaften T, et al. Stem cell conditioned medium improves acute lung injury in mice: in vivo evidence for stem cell paracrine action. Am J Physiol Lung Cell Mol Physiol 2012;303: L967-77.

44. Hodges RJ, Jenkin G, Hooper SB, et al. Human amnion epithelial cells reduce ventilation-induced preterm lung injury in fetal sheep. Am J Obstet Gynecol 2012;206:448 e8-15.

45. Vosdoganes P, Hodges RJ, Lim R, et al. Human amnion epithelial cells as a treatment for inflammation-induced fetal lung injury in sheep. Am J Obstet Gynecol 2011;205:156 e26-33.

46. Di Nicola M, Carlo-Stella C, Magni M, et al. Human bone marrow stromal cells suppress T-lymphocyte proliferation induced by cellular or nonspecific mitogenic stimuli. Blood 2002;99:3838-43.

47. Gupta N, Su X, Popov B, Lee JW, Serikov V, Matthay MA. Intrapulmonary delivery of bone marrow-derived mesenchymal stem cells improves survival and attenuates endotoxin-induced acute lung injury in mice. J Immunol 2007;179:1855-63.

48. Chang YS, Ahn SY, Jeon HB, et al. Critical role of VEGF secreted by mesenchymal stem cells in hyperoxic lung injury. Am J Respir Cell Mol Biol 2014;51:391-9.

49. Chen Y, Qian H, Zhu W, et al. Hepatocyte growth factor modification promotes the amelioration effects of human umbilical cord mesenchymal stem cells on rat acute kidney injury. Stem Cells Dev 2011;20:103-3.

50. Curley GF, Hayes M, Ansari B, et al. Mesenchymal stem cells enhance recovery and repair following ventilator-induced lung injury in the rat. Thorax 2012;67:496-501.

51. Mahmood A, Lu D, Chopp M. Intravenous administration of marrow stromal cells (MSCs) increases the expression of growth factors in rat brain after traumatic brain injury. J Neurotrauma 2004;21: 33-9.

52. Imberti $\mathrm{B}$, Morigi $\mathrm{M}$, Tomasoni $\mathrm{S}$, et al. Insulin-like growth factor-1 sustains stem cell mediated renal repair. J Am Soc Nephrol 2007;18: 2921-8.

53. Fang X, Neyrinck AP, Matthay MA, Lee JW. Allogeneic human mesenchymal stem cells restore epithelial protein permeability in cultured human alveolar type II cells by secretion of angiopoietin-1. J Biol Chem 2010;285:26211-2.

54. Danchuk S, Ylostalo JH, Hossain F, et al. Human multipotent stromal cells attenuate lipopolysaccharide-induced acute lung injury in mice via secretion of tumor necrosis factor-alpha-induced protein 6. Stem Cell Res Ther 2011;2:27.

55. Ortiz LA, Dutreil M, Fattman C, et al. Interleukin 1 receptor antagonist mediates the antiinflammatory and antifibrotic effect of mesenchymal stem cells during lung injury. Proc Natl Acad Sci USA 2007;104: 11002-7.

56. Gupta N, Krasnodembskaya A, Kapetanaki M, et al. Mesenchymal stem cells enhance survival and bacterial clearance in murine Escherichia coli pneumonia. Thorax 2012;67:533-9.

57. Krasnodembskaya A, Song Y, Fang X, et al. Antibacterial effect of human mesenchymal stem cells is mediated in part from secretion of the antimicrobial peptide LL-37. Stem Cells 2010;28:2229-38.

58. Sung DK, Chang YS, Sung SI, Yoo HS, Ahn SY, Park WS. Antibacterial effect of mesenchymal stem cells against Escherichia coli is mediated by secretion of beta- defensin- 2 via toll- like receptor 4 signalling. Cell Microbiol 2016;18:424-36.

59. Fang X, Abbott J, Cheng L, et al. Human mesenchymal stem (stromal) cells promote the resolution of acute lung injury in part through lipoxin A4. J Immunol 2015;195:875-1.
60. Tsoyi K, Hall SR, Dalli J, et al. Carbon monoxide improves efficacy of mesenchymal stromal cells during sepsis by production of specialized proresolving lipid mediators. Crit Care Med 2016;44:e1236-45.

61. Pluchino S, Cossetti C. How stem cells speak with host immune cells in inflammatory brain diseases. Glia 2013;61:1379-401.

62. Sdrimas K, Kourembanas S. MSC microvesicles for the treatment of lung disease: a new paradigm for cell-free therapy. Antioxid Redox Signal 2014;21:1905-5.

63. Lee C, Mitsialis SA, Aslam M, et al. Exosomes mediate the cytoprotective action of mesenchymal stromal cells on hypoxia-induced pulmonary hypertension. Circulation 2012;126:2601-11.

64. Ophelders DR, Wolfs TG, Jellema RK, et al. Mesenchymal stromal cellderived extracellular vesicles protect the fetal brain after hypoxiaischemia. Stem Cells Transl Med 2016;5:754-63.

65. Mokarizadeh A, Delirezh N, Morshedi A, Mosayebi G, Farshid AA, Mardani K. Microvesicles derived from mesenchymal stem cells: potent organelles for induction of tolerogenic signaling. Immunol Lett 2012;147:47-54.

66. Zhou Y, Xu H, Xu W, et al. Exosomes released by human umbilical cord mesenchymal stem cells protect against cisplatin-induced renal oxidative stress and apoptosis in vivo and in vitro. Stem Cell Res Ther 2013;4:34.

67. Yu B, Kim HW, Gong M, et al. Exosomes secreted from GATA-4 overexpressing mesenchymal stem cells serve as a reservoir of antiapoptotic microRNAs for cardioprotection. Int J Cardiol 2015;182: $349-60$.

68. Willis GR, Fernandez-Gonzalez A, Anastas J, et al. Mesenchymal stromal cell exosomes ameliorate experimental bronchopulmonary dysplasia and restore lung function through macrophage immunomodulation. Am J Respir Crit Care Med 2017 (e-pub ahead of print).

69. Rager TM, Olson JK, Zhou Y, Wang Y, Besner GE. Exosomes secreted from bone marrow-derived mesenchymal stem cells protect the intestines from experimental necrotizing enterocolitis. J Pediatr Surg 2016;51:942-7.

70. Heldring N, Mager I, Wood MJ, Le Blanc K, Andaloussi SE. Therapeutic potential of multipotent mesenchymal stromal cells and their extracellular vesicles. Hum Gene Ther 2015;26:506-17.

71. Katsuda T, Ochiya T. Molecular signatures of mesenchymal stem cellderived extracellular vesicle-mediated tissue repair. Stem Cell Res Ther 2015;6:212.

72. Kourembanas S. Exosomes: vehicles of intercellular signaling, biomarkers, and vectors of cell therapy. Annu Rev Physiol 2015;77: $13-27$.

73. Borger V, Bremer M, Ferrer-Tur R, et al. Mesenchymal stem/stromal cell-derived extracellular vesicles and their potential as novel immunomodulatory therapeutic agents. Int J Mol Sci 2017;18.

74. Hayes M, Curley GF, Masterson C, Devaney J, O'Toole D, Laffey JG. Mesenchymal stromal cells are more effective than the MSC secretome in diminishing injury and enhancing recovery following ventilatorinduced lung injury. Intens Care Med Exp 2015;3:29.

75. Islam MN, Das SR, Emin MT, et al. Mitochondrial transfer from bonemarrow-derived stromal cells to pulmonary alveoli protects against acute lung injury. Nat Med 2012;18:759-65.

76. Li X, Zhang Y, Yeung SC, et al. Mitochondrial transfer of induced pluripotent stem cell-derived mesenchymal stem cells to airway epithelial cells attenuates cigarette smoke-induced damage. Am J Respir Cell Mol Biol 2014;51:455-65.

77. Jackson MV, Morrison TJ, Doherty DF, et al. Mitochondrial transfer via tunneling nanotubes is an important mechanism by which mesenchymal stem cells enhance macrophage phagocytosis in the in vitro and in vivo models of ARDS. Stem Cells 2016;34:2210-3.

78. Phinney DG, Di Giuseppe M, Njah J, et al. Mesenchymal stem cells use extracellular vesicles to outsource mitophagy and shuttle microRNAs. Nat Commun 2015;6:8472.

79. Morrison TJ, Jackson MV, Cunningham EK, et al. Mesenchymal stromal cells modulate macrophages in clinically relevant lung injury models by 


\section{Enhancing paracrine potency of MSCs $\quad$ Review}

extracellular vesicle mitochondrial transfer. Am J Respir Crit Care Med 2017 (e-pub ahead of print).

80. Ankrum JA, Ong JF, Karp JM. Mesenchymal stem cells: immune evasive, not immune privileged. Nat Biotechnol 2014;32:252-60.

81. Mitsialis SA, Kourembanas S. Stem cell-based therapies for the newborn lung and brain: possibilities and challenges. Semin Perinatol 2016;40: $138-51$.

82. Doorn J, Moll G, Le Blanc K, van Blitterswijk C, de Boer J. Therapeutic applications of mesenchymal stromal cells: paracrine effects and potential improvements. Tissue Eng Part B Rev 2012;18:101-5.

83. Hasty KA, Cho H. Stem cell considerations for the clinician. Phys Med Rehabil Clin N Am 2016;27:855-70.

84. Mueller M, Kramer BW. Stem cells and bronchopulmonary dysplasiathe five questions: which cells, when, in which dose, to which patients via which route? Paediatr Respir Rev 2016;24:54-9.

85. Prockop DJ, Kota DJ, Bazhanov N, Reger RL. Evolving paradigms for repair of tissues by adult stem/progenitor cells (MSCs). J Cell Mol Med 2010;14:2190-9.

86. Murphy MB, Moncivais K, Caplan AI. Mesenchymal stem cells: environmentally responsive therapeutics for regenerative medicine. Exp Mol Med 2013;45:e54.

87. Caplan AI. MSCs: the sentinel and safe-guards of injury. J Cell Physiol 2016;231:1413-6.

88. Waterman RS, Tomchuck SL, Henkle SL, Betancourt AM. A new mesenchymal stem cell (MSC) paradigm: polarization into a proinflammatory MSC1 or an Immunosuppressive MSC2 phenotype. PLoS ONE 2010;5:e10088.

89. Bernardo ME, Fibbe WE. Mesenchymal stromal cells: sensors and switchers of inflammation. Cell Stem Cell 2013;13:392-402.

90. Auletta JJ, Bartholomew AM, Maziarz RT, et al. The potential of mesenchymal stromal cells as a novel cellular therapy for multiple sclerosis. Immunotherapy 2012;4:529-47.

91. Balan A, Lucchini G, Schmidt S, et al. Mesenchymal stromal cells in the antimicrobial host response of hematopoietic stem cell recipients with graft-versus-host disease-friends or foes? Leukemia 2014;28: 1941-8.

92. van Velthoven CT, Kavelaars A, Heijnen CJ. Mesenchymal stem cells as a treatment for neonatal ischemic brain damage. Pediatr Res 2012;71: $474-81$.

93. Caplan AI. Mesenchymal stem cells: time to change the name!. Stem Cells Transl Med 2017;6:1445-51.

94. Chang YS, Ahn SY, Sung S, Park WS. Stem cell therapy for neonatal disorders: prospects and challenges. Yonsei Med J 2017;58:266-71.

95. Ahn SY, Chang YS, Park WS. Stem cells for neonatal brain disorders. Neonatology 2016;109:377-83.

96. Dominici M, Le Blanc K, Mueller I, et al. Minimal criteria for defining multipotent mesenchymal stromal cells. The International Society for Cellular Therapy position statement. Cytotherapy 2006;8: 315-7.

97. Hass R, Kasper C, Bohm S, Jacobs R. Different populations and sources of human mesenchymal stem cells (MSC): a comparison of adult and neonatal tissue-derived MSC. Cell Commun Signal 2011;9:12.

98. Mendicino M, Bailey AM, Wonnacott K, Puri RK, Bauer SR. MSC-based product characterization for clinical trials: an FDA perspective. Cell Stem Cell 2014;14:141-5.

99. Pittenger MF, Mackay AM, Beck SC, et al. Multilineage potential of adult human mesenchymal stem cells. Science 1999;284:143-7.

100. Pacini S. Deterministic and stochastic approaches in the clinical application of mesenchymal stromal cells (MSCs). Front Cell Dev Biol 2014;2:50.

101. Mobius MA, Thebaud B. Stem cells and their mediators-next generation therapy for bronchopulmonary dysplasia. Front Med (Lausanne) 2015;2:50.

102. Galipeau J, Krampera M. The challenge of defining mesenchymal stromal cell potency assays and their potential use as release criteria. Cytotherapy 2015;17:125-7.
103. Kebriaei P, Isola L, Bahceci E, et al. Adult human mesenchymal stem cells added to corticosteroid therapy for the treatment of acute graftversus-host disease. Biol Blood Marrow Transplant 2009;15:804-11.

104. Jiao J, Milwid JM, Yarmush ML, Parekkadan B. A mesenchymal stem cell potency assay. Methods Mol Biol 2011;677:221-31.

105. Kern S, Eichler H, Stoeve J, Kluter H, Bieback K. Comparative analysis of mesenchymal stem cells from bone marrow, umbilical cord blood, or adipose tissue. Stem Cells 2006;24:1294-301.

106. Amable PR, Teixeira MV, Carias RB, Granjeiro JM, Borojevic R. Protein synthesis and secretion in human mesenchymal cells derived from bone marrow, adipose tissue and Wharton's jelly. Stem Cell Res Ther 2014;5: 53.

107. Pievani A, Scagliotti V, Russo FM, et al. Comparative analysis of multilineage properties of mesenchymal stromal cells derived from fetal sources shows an advantage of mesenchymal stromal cells isolated from cord blood in chondrogenic differentiation potential. Cytotherapy 2014;16:893-905.

108. Schafer R, Spohn G, Baer PC. Mesenchymal stem/stromal cells in regenerative medicine: can preconditioning strategies improve therapeutic efficacy? Transfus Med Hemother 2016;43:256-67.

109. Ji ST, Kim H, Yun J, Chung JS, Kwon SM. Promising therapeutic strategies for mesenchymal stem cell-based cardiovascular regeneration: from cell priming to tissue engineering. Stem Cells Int 2017;2017: 3945403.

110. Waszak P, Alphonse R, Vadivel A, Ionescu L, Eaton F, Thebaud B. Preconditioning enhances the paracrine effect of mesenchymal stem cells in preventing oxygen-induced neonatal lung injury in rats. Stem Cells Dev 2012;21:2789-97.

111. Danielyan L, Schafer R, Schulz A, et al. Survival, neuron-like differentiation and functionality of mesenchymal stem cells in neurotoxic environment: the critical role of erythropoietin. Cell Death Differ 2009;16:1599-614.

112. Kang S, Kim SM, Sung JH. Cellular and molecular stimulation of adipose-derived stem cells under hypoxia. Cell Biol Int 2014;38:553-62.

113. Ceradini DJ, Kulkarni AR, Callaghan MJ, et al. Progenitor cell trafficking is regulated by hypoxic gradients through HIF-1 induction of SDF-1. Nat Med 2004;10:858-64.

114. Bruno S, Grange C, Collino F, et al. Microvesicles derived from mesenchymal stem cells enhance survival in a lethal model of acute kidney injury. PLoS ONE 2012;7:e33115.

115. Amin AH, Abd Elmageed ZY, Nair D, et al. Modified multipotent stromal cells with epidermal growth factor restore vasculogenesis and blood flow in ischemic hind-limb of type II diabetic mice. Lab Invest 2010;90:985-6.

116. Rota C, Imberti B, Pozzobon M, et al. Human amniotic fluid stem cell preconditioning improves their regenerative potential. Stem Cells Dev 2012;21:1911-23.

117. Xinaris C, Morigi M, Benedetti V, et al. A novel strategy to enhance mesenchymal stem cell migration capacity and promote tissue repair in an injury specific fashion. Cell Transplant 2013;22:423-36.

118. Heo SC, Jeon ES, Lee IH, Kim HS, Kim MB, Kim JH. Tumor necrosis factor-alpha-activated human adipose tissue-derived mesenchymal stem cells accelerate cutaneous wound healing through paracrine mechanisms. J Invest Dermatol 2011;131:1559-67.

119. Pasha Z, Wang Y, Sheikh R, Zhang D, Zhao T, Ashraf M. Preconditioning enhances cell survival and differentiation of stem cells during transplantation in infarcted myocardium. Cardiovasc Res 2008;77:134-42.

120. Tang Y, Cai B, Yuan F, et al. Melatonin pretreatment improves the survival and function of transplanted mesenchymal stem cells after focal cerebral ischemia. Cell Transplant 2014;23:1279-91.

121. Yao Y, Zhang F, Wang L, et al. Lipopolysaccharide preconditioning enhances the efficacy of mesenchymal stem cells transplantation in a rat model of acute myocardial infarction. J Biomed Sci 2009;16:74.

122. Zhang J, Chen GH, Wang YW, et al. Hydrogen peroxide preconditioning enhances the therapeutic efficacy of Wharton's Jelly 
mesenchymal stem cells after myocardial infarction. Chin Med J (Engl) 2012;125:3472-8.

123. Najafi R, Sharifi AM. Deferoxamine preconditioning potentiates mesenchymal stem cell homing in vitro and in streptozotocindiabetic rats. Expert Opin Biol Ther 2013;13:959-72.

124. Cui X, Wang H, Guo H, et al. Transplantation of mesenchymal stem cells preconditioned with diazoxide, a mitochondrial ATP-sensitive potassium channel opener, promotes repair of myocardial infarction in rats. Tohoku J Exp Med 2010;220:139-47.

125. Beegle J, Lakatos K, Kalomoiris S, et al. Hypoxic preconditioning of mesenchymal stromal cells induces metabolic changes, enhances survival, and promotes cell retention in vivo. Stem Cells 2015;33:1818-28.

126. Goodman RB, Pugin J, Lee JS, Matthay MA. Cytokine-mediated inflammation in acute lung injury. Cytokine Growth Factor Rev 2003;14:523-35.

127. Kurozumi K, Nakamura K, Tamiya T, et al. Mesenchymal stem cells that produce neurotrophic factors reduce ischemic damage in the rat middle cerebral artery occlusion model. Mol Ther 2005;11:96-104.

128. van Velthoven CT, Sheldon RA, Kavelaars A, et al. Mesenchymal stem cell transplantation attenuates brain injury after neonatal stroke. Stroke 2013;44:1426-32.

129. Tenzen T, Zembowicz F, Cowan CA. Genome modification in human embryonic stem cells. J Cell Physiol 2010;222:278-81.

130. Rojas $\mathrm{M}, \mathrm{Xu} J$, Woods CR, et al. Bone marrow-derived mesenchymal stem cells in repair of the injured lung. Am J Respir Cell Mol Biol 2005;33:145-52.

131. Reyes B, Coca MI, Codinach M, et al. Assessment of biodistribution using mesenchymal stromal cells: algorithm for study design and challenges in detection methodologies. Cytotherapy 2017;19:1060-9.

132. Park WS, Sung SI, Ahn SY, et al. Optimal timing of mesenchymal stem cell therapy for neonatal intraventricular hemorrhage. Cell Transplant 2016;25:1131-44.
133. Kim ES, Ahn SY, Im GH, et al. Human umbilical cord blood-derived mesenchymal stem cell transplantation attenuates severe brain injury by permanent middle cerebral artery occlusion in newborn rats. Pediatr Res 2012;72:277-84

134. Deuse T, Peter C, Fedak PW, et al. Hepatocyte growth factor or vascular endothelial growth factor gene transfer maximizes mesenchymal stem cell-based myocardial salvage after acute myocardial infarction. Circulation 2009;120:S247-54.

135. Kokaia Z, Martino G, Schwartz M, Lindvall O. Cross-talk between neural stem cells and immune cells: the key to better brain repair? Nat Neurosci 2012;15:1078-87.

136. Donega V, van Velthoven $\mathrm{CT}$, Nijboer $\mathrm{CH}$, et al. Intranasal mesenchymal stem cell treatment for neonatal brain damage: longterm cognitive and sensorimotor improvement. PLoS ONE 2013;8: e51253.

$($ ()) This work is licensed under a Creative Commons Attribution-NonCommercial-NoDerivs 4.0

International License. The images or other third party material in this article are included in the article's Creative Commons license, unless indicated otherwise in the credit line; if the material is not included under the Creative Commons license, users will need to obtain permission from the license holder to reproduce the material. To view a copy of this license, visit http://creativecommons.org/licenses/ by-nc-nd/4.0/

(C) The Author(s) (2018) 